\title{
AN INTERACTIVE CONCATENATED TURBO CODING SYSTEM
}

\section{Ye Liu, Heng Tang, Shu Lin and Marc Fossorier}


工 


\title{
AN INTERACTIVE CONCATENATED TURBO CODING SYSTEM*
}

\author{
Ye Liu, Heng Tang, Shu Lin and Marc Fossorier \\ Department of Electrical Engineering \\ University of Hawaij at Manoa
}

September 13, 1999

\begin{abstract}
This paper presents a concatenated turbo coding system in which a Reed-Solomon outer code is concatenated with a binary turbo innet code. In the proposed system, the outer code decoder and the inner turbo code decoder interact to achieve both good bit ertor and frame error performances. The outer code decoder helps the inner turbo code decoder to terminate its decoding iteration while the inner turbo code decoder provides soft-output information to the outer code decoder to carty out a reliability-based softdecision decoding. In the case that the outer code decoding fails, the outer code decoder instructs the inner code decoder to continue its decoding iterations until the outer code decoding is successful or a preset maximnm number of decoding iterations is reached. This interaction betreen outer and inner code decoders reduces decoding delay. Also presented in the paper are an effective criterion for stopping the iteration process of the inner code decoder and a new reliability-based decoding algorithm for nonbinary codes.
\end{abstract}

-This research was supported by NSF under Grants NCR 94-15374, CCR 97-32959, CCR 98-14054 and NASA under Grant NAG 5-931. 


\section{Introduction}

Although turbo codes with iterative decoding $(1,2,3)$ have been shown to achieve bit-error rates (BER's) of $10^{-3}$ or better at SNR's within $1 \mathrm{~dB}$ of the SNR for which the code rate equals channel capacity, they suffer from three disadvantages: (1) a large decoding delay due to the large block lengths and many decoding iterations required for near capacity performance, (2) significant weakened performance at BER's below $10^{-5}$ due to the fact that the component codes bave relatively poor minimum distances, which manifests itself at very low BER's, and (3) a relatively poor frame error performance. The large decoding delay makes turbo codes unsuilable for real time applications such as voice transmission and packet communications in high speed networks. The fact that turbo codes do not have large minimum distances causes the BER curve to fatten out at BER's below $10^{-5}$. This phenomenon is called error floor. Because of the error floor, turbo codes are not suitable for applications requiring extremely low BER's, such as some scientific or command and control applications. Poor frame error performance is due to the fact that turbo decoding is devised to minimize bit error probability not the frame error probability. Even though a decoded block may contain very few errors, it is still an erroneous block. Poor frame error performance also makes these codes not suitable for many communication applications where reliable frame transmission is required. There are measures that can be taken to mitigate the error foor and poor frame error performance problems. One such measure is to use a powerful Reed-Solomon (RS) outer code in concatenation with a turbo inner code in a proper way.

In this paper, we present an interactive concatenated turbo coding system in which an RS outer code is concatenated with a high rate binary turbo inner code, and the outer code decoder and the inner turbo code decoder interact to achieve both good bit-error and frameerror performances. The inner turbo decoder consists of two component decoders which 
operate in parallel mode. The two component decoders process their inputs simultaneously. At the completion of a decoding phase, their decoded outputs (log.likehood ratios and hard. decisions of the decoded binary symbols) are compared. When the comparison satisfies a certain criterion, the inner turbo decoder stops its decoding iteration and the outer code decoder takes over and completes the decoding process. If the outer code decoding is not successful (i.e., a decoding failure), the outer code decoder instructs the inner turbo decoder to continue its decoding iterations until the symbol errors at the input of the outer decoder is reduced within the error correction capability of the outer code. The interactive process continues until either the outer decoding is successful or a preset maximum number of decoding iterations for the inner turbo decoder is rearched. In the latter case, the outer code decoder computes the reliability values of its input symbols based on the soft output information (log-likebood ratios of the decoded bits) of inner turbo code decoder and carries out a reliability-based soft-decision decoding algorithm.

Also presented in this paper are a new stopping criterion for the inner turbo decoding and a new reliability-based algorithm for decoding nonbinary block codes. The new stopping criterion with the aid of outer code decoding effectively terminates the turbo decoding process with negligible degradation in error performance compared with the cross entropy (CE) stopping criterion [3]. It provides a significant reduction in the number of decoding iterations and hence reduces decoding delay. The new reliability-based decoding algorithm is devised by combining the Chase-2 decoding algorithm [4] and the generalized minimum distance (GMD) decoding algorithm [5]. This decoding algorithm provides a good trade-off between error performance of the Chase-2 algorithm and decoding complexity of the GMD algorithm.

Simulation results show that the proposed concatenated turbo coding system with the new stopping criterion for the inner turbo decoding and the new reliability-based algorithm for decoding the outer RS code achieves both good bit error and frame error performances and reduces decoding delay. 


\section{Turbo Codes, Parallel Turbo Decoding and Bit Matching Stopping Criterion}

A turbo code with linear block codes as component codes is obtained by parallel concatenation of two systematic linear block codes with a pseudo random interlever $\Pi$ between two encoders as shown in Figure 1. Assume that two component codes are identical and both are $\left(n_{i}, k_{i}\right)$ binary linear block codes. Let $u=\left(u_{0}, u_{1}, \cdots, u_{K-1}\right)$ be the information sequence to be encoded for transmission where $K=1 k_{i}$. The first encoder encodes this sequence and produces a block of $l\left(n_{i}-k_{i}\right)$ parity-check bits, denoted $\mathrm{p}^{(1)}$. The interlever $I 1$ permutes the information sequence $u$ into a sequence $u^{\prime}=\Pi(u)$. The second encoder encodes $u^{\prime}$ and produces a block of $l\left(n_{i}-k_{1}\right)$ parity-check bits, denoted $p^{(2)}$. Then the sequence $\left(u, p^{(1)}, p^{(2)}\right)$ is the code sequence for the information sequence $u$. The collection of $2^{K}$ such code sequences, one for each information sequence $u$, form a turbo code of length $N=1\left(2 n_{i}-k_{i}\right)$. Since the component codes are block codes, it is called a block turbo code.

The decoder for a turbo code with two component codes consists of two soft-input/softoutput (SISO) MAP (or APP) decoders which operate iteratively $[1,2,3]$. Since the two component codes are identical, the two MAP decoders are identical. Decoding can be carried out in either serial mode or parallel mode as shown in Figures 2(a) and 2(b), respectively. The serial decoding mode was originally proposed by Berrou et. al. [1], and the parallel decoding mode was later proposed by Divsalar and Pollara [6]. In serial mode, decoder 1 and decoder 2 , denoted DEC1 and DEC2, respectively, operates alternately. In parallel mode, the two MAP decoders, DEC1 and DEC2, operate simultaneously. Decoding consists of a sequence of iterations, each decoding iteration consists of two phases. In serial mode, DECl operates in the first phase and DEC2 operates in the second phase as shown in Figure 2(a). However in parallel mode, both DEC1 and DEC2 operates in each phase as shown in Figure 2(b). 
In the proposed interactive concatenated turbo coding system, inner turbo decoding is performed in parallel mode. Suppose a code sequence $\left(u, p^{(1)}, p^{(2)}\right)$ is transmitted. Let $r=\left(r_{0}, r_{1}, \cdots, r_{N-1}\right)$ be the received sequence. For decoding, this received sequence is de. composed into two subsequences $r^{(1)}=\left(r_{0}^{(1)}, r_{1}^{(1)}, \cdots\right)$ and $r^{(2)}=\left(r_{0}^{(2)}, r_{1}^{(2)}, \cdots\right)$, corresponding to code sequences $\left(u, p^{(1)}\right)$ and $\left(u^{\prime}, p^{(2)}\right)$ at the outputs of two component encoders, respectively. Each SISO decoder bas two inputs and two outputs as shown in Figure 2(c). The inputs to each decoder are the a priori $L$-values (log-likelihood values) $L\left(u_{i}\right)$ for all information bits $u_{1}$ and the received channel $L$-values $L_{c} r_{1}$ for all code bits, where $L_{c}=4 a E_{v} / N_{0}$ and $E_{J} / N_{0}$ is the channel SNR. For a fading channel, a denotes the fading amplitude, whereas for an AWGN channel, $a=1$. Based on its inputs, the SISO decoder computes $L$-values (soft outputs)

$$
L\left(\dot{u}_{i}\right) \triangleq L\left(u_{i} \mid \mathbf{r}\right)=\log \frac{p\left(u_{i}=1 \mid \mathbf{r}\right)}{p\left(u_{i}=0 \mid \mathbf{r}\right)},
$$

for all information bits and delivers an extrinsic $L$-value $L_{\varepsilon}\left(\dot{u}_{i}\right)$ for each information bit which contains the reliability information from all other coded bits in the code sequence and is not influenced by $L\left(u_{i}\right)$ and $L_{c} r_{i}$ of the current bit $u_{i}$.

Consider turbo decoding in parallel mode. At the first iteration, DECl and DEC 2 start decoding at the same time. The inputs to DECl and DEC2 are channel $L$-values $L_{c} r_{i}^{(1)}$ and $L_{c} r_{i}^{(2)}$, respectively. For equally likely information bits, the a priori $L$-value $L\left(u_{i}\right)$ inputs to both SISO decoders in the first phase of the first iteration are zero. Hence, we set $L^{(1)}\left(u_{i}\right)=0$ and $L^{(2)}\left(u_{i}\right)=0$ for each information bit. The outputs of DEC1 and DEC2 are $L$-values $L^{(1)}\left(\hat{u}_{i}\right)$ and $L^{(2)}\left(\hat{u}_{i}\right)$ and extrinsic $L$-values $L_{e}^{(1)}\left(\hat{u}_{i}\right)$ and $L_{e}^{(2)}\left(\hat{u}_{i}\right)$, respectively, with $0 \leq i<K$. Then the second phase starts. The inputs to DECl are channel $L$-values $L_{c} \mathbf{r}^{(1)}$ and extrinsic values $L_{e}^{(2)}\left(\hat{u}_{i}\right)$ 's which are the outputs of DEC2 in the first decoding phase. The inputs to DEC2 are $L_{c} \mathbf{r}^{(2)}$ and $L_{\varepsilon}^{(1)}\left(\hat{u}_{i}\right)$ 's which are the outputs of DEC1 in the first decoding phase. The second decoding phase is then performed. All the subsequent iterations are carried out in the same manner as the first iteration except that the a priori $L$-values 
$L^{(1)}\left(u_{i}\right)$ and $L^{(2)}\left(u_{i}\right)$ of each information bit to the inputs of the two SISO decoders at the first decoding phase are the extrinsic $L$-values $L_{c}^{(2)}\left(\dot{u}_{i}\right)$ and $L_{c}^{(1)}\left(\dot{u}_{i}\right)$, respectively, which are the outputs of the two decoders in the second decoding phase of the previous iteration.

After a sufficient number of iterations (or decoding phases), we can stop the decoding process and obtain the $L$-value for each information bit as follow:

$$
L\left(\hat{u}_{i}\right)= \begin{cases}L^{(1)}\left(\dot{u}_{i}\right), & \text { if }\left|L^{(1)}\left(\dot{u}_{i}\right)\right| \geq\left|L^{(2)}\left(\dot{u}_{i}\right)\right|, \\ L^{(2)}\left(\dot{u}_{i}\right), & \text { otherwise, }\end{cases}
$$

where $L^{(1)}\left(\dot{u}_{i}\right)=L_{c} \cdot r_{i}^{(1)}+L^{(1)}\left(u_{i}\right)+L_{c}^{(1)}\left(\dot{u}_{i}\right)$, and $L^{(2)}\left(\dot{u}_{i}\right)=L_{c} \cdot r_{i}^{(2)}+L^{(2)}\left(u_{i}\right)+L_{c}^{(2)}\left(\dot{u}_{i}\right)$. Finally, the hard-decision decoded information bit $u_{i}$ is made based on

$$
\dot{u}_{i}= \begin{cases}0, & \text { if } L\left(\dot{u}_{i}\right) \leq 0 \\ 1, & \text { if } L\left(\dot{u}_{i}\right)>0,\end{cases}
$$

for $0 \leq i<K$.

As the iterative decoding approaches the performance limit of a given turbo code, any further iteration results in very little improvement in performance. Therefore it is important to devise an efficient criterion to stop the the iteration process and prevent unnecessary computations and decoding delay. Several stopping criteria bave been devised $[3,7]$. Both the sign change and bit matching criteria proposed in [7] are more computationally efficient than the cross entropy (CE) criterion proposed in [3].

The bit matching (BM) criterion of [7] can be applied to terminate decoding in parallel mode in a straightforward manner. At the $j$-th decoding phase of the $k$-th iteration for $j=1,2$ and $k=1,2, \cdots$, we check the hard decisions based on the $L$-values $L^{(1)}\left(\hat{u}_{i}\right)$ and $L^{(2)}\left(\hat{u}_{i}\right)$ generated by $\mathrm{DECl}$ and DEC2, respectively, for each information bit. If these bard decisions agree with each other for all the information bits in the whole sequence, we terminate the decoding process at the $j$-th phase of the $k$-th iteration.

At each phase, the BM criterion requires $2 K$ binary operations to make hard decisions based on $L^{(1)}\left(\hat{u}_{i}\right)$ and $L^{(2)}\left(\hat{u}_{i}\right)$ and $K$ logic operations to check whether the BM criterion is 
satisfied. However to test the CE criterion at each iteration, it requires a total of $5 K-1$ real number operations, including $2 K-1$ additions and subtractions, $2 K$ multiplications and divisions, and $K$ exponentiations. Therefore, the $\mathrm{BM}$ criterion requires much simpler computations than the $\mathrm{CE}$ criterion.

Simulation results show that the $\mathrm{BM}$ criterion saves more iterations than the $\mathrm{CE}$ criterion with negligible degradation in error performance. Consider the turbo code with the $(64,57)$ distance-4 extended Hamming code as the two component codes and a block interleaver of size $K=57 \times 57$. The error performances of decoding in parallel mode with BM stopping criterion and serial mode with CE stopping criteria are shown in Figure 3(a). We see that decoding in parallel mode with BM stopping criterion outperforms decoding in serial mode with $\mathrm{CE}$ stopping criterion. The average numbers of decoding iterations required using BMI and $\mathrm{CE}$ criteria, respectively, for parallel mode decoding of the above turbo code are shown in Figure 3(b). We see that the BM stopping criterion saves more decoding iterations than the $\mathrm{CE}$ stopping criterion and bence reduces computational complexity. From Figure 3(a), we also see that the frame error performance is relatively poor compared with the bit error performance. The error floor starts at frame error probability of $10^{-2}$. This error floor will be removed when the proposed concatenated turbo system is used.

\section{Chase-GMD Decoding Algorithm}

RS codes are commonly decoded with an algebraic decoding algorithm, such as the Euclidean Algorithm, in applications for keeping the decoding complexity low. To improve the error performance, soft-decision decoding must be used. However, soft-decision decoding of RS codes significantly increases the decoding complexity. One approach to improve the performance of algebraic decoding while keeping low decoding complexity is to use an algebraic decoder to generate a sequence of candidate codewords based on the reliability values of 
the received symbols, and then choose the candidale codeword with the best metric as the decoded codeword. The two most well known sucb decoding algorithms are GMD algorithm [5] and Chase-2 algorithm [4]. Both algorithms improve the error performance of algebraic decoding. For a RS code over $\mathrm{GF}(q)$ with minimum distance $d, \mathrm{GMD}$ algorithm requires to perform at most $\lfloor(d+1) / 2\rfloor$ algebraic decodings while Chase. 2 algorithm needs to perform $q^{[d / 2]}$ algebraic decodings based on $q^{[d / 2]}$ test error patterns with errors confined to $[d / 2\rfloor$ least reliable positions of the received sequence. Chase- 2 algorithm outperforms GMD al. gorithm, however it requires much more decoding computations. For long RS codes over large field $G F(q)$ with large minimum distance $d$, Chase. 2 algorithm becomes impractical. The GMD decoding algorithm while simple gives only small improvement in error performance over pure algebraic decoding for small to medium SNR's, especially for long RS codes. Therefore, GMD is not very attractive for practical applications for small to medium SNR's and therefore, it must be improved.

In this section, we present a decoding algorithm which combines Chase-2 and GMD algorithms. It provides a good trade-off between the error performance of Chase- 2 algorithm and the decoding complexity of GMD algorithm. We call this decoding algorithm ChaseGMD algorithm.

Consider an $\left(n_{0}, k_{0}, d\right)$ RS code over GF( $\left.q\right)$ with $q=2^{m}$. Let $x=\left(x_{0}, x_{1}, \cdots, x_{n_{0}-1}\right)$ be a codeword. For binary transmission, every code symbol $x_{i}$ is expanded into a binary $m$ tuple. Let $y=\left(y_{0}, y_{1}, \cdots, y_{n_{0}-1}\right)$ be the unquantized received sequence at the output of the matched filter in the receiver, where $y_{i}$ represents a vector $\left(y_{i, 0}, y_{i, 1}, \cdots, y_{i, m-1}\right)$ composed of $m$ real numbers. Let $z=\left(z_{0}, z_{1}, \cdots, z_{n_{0}-1}\right)$ be the hard-decision received sequence obtained from $y$ with $z_{i}$ in $G F\left(2^{m}\right)$. A real number $\alpha_{i}$ is assigned to each hard-decision received symbol $z_{i}$ to indicate its reliability. There are a number of ways to define $\alpha_{i}$ 's $[5,8]$. For the proposed concatenated turbo coding system, since inner turbo decoding not only gives the hard-decision of each information bit but also provides its reliability $L$-value. Based on 
the bit reliability values, we can easily compute the reliability value of each hard-decision received symbol $z_{i}$. Let $\left(x_{i, 0}, x_{i, 1}, \cdots, x_{i, m-1}\right)$ be the binary $m$-tuple expansion of code symbol $x_{i}$. With respect to inner encoding, each $x_{i j}$ witb $0 \leq j<m$, is an information bit. The inner turbo decoding provides each bit $x_{i j}$ a reliability $L$-value, $L\left(\dot{x}_{i j}\right)$. Then the reliability value of the $i$-th hard-decision received symbol $z_{i}$ is

$$
\alpha_{i}=\prod_{j=0}^{m-1} p\left(\dot{i}_{i j}\right)
$$

where

$$
p\left(\hat{x}_{i,}= \pm 1\right)=\frac{e^{ \pm L\left(\dot{x}_{i, j}\right)}}{1+\epsilon^{ \pm L\left(\dot{x}_{1, j}\right)}}
$$

for $0 \leq i<n_{0}$ and $0 \leq j<m$. The larger $\alpha_{i}$, the more reliable $z_{i}$ is.

Now we describe the Chase-GMD algorithm. Witbout loss of generality, we assume that the hard-decision received symbols in $z$ are ordered in the order of increasing reliability such that $\alpha_{i} \leq \alpha_{j}$ for $i<j$. We also assume that an error-and-erasure algebraic decoder [5] is used to generate candidate codewords which corrects e errors and $s$ erasures provided that $s+2 e<d$. For $0 \leq P \leq\lfloor d / 2\rfloor$, let $E$ denote the set of $q^{P}$ test error patterns with errors (nonzero components) confined to the $P$ least reliable positions. Let $\operatorname{CGA}(P)$ denote the Chase-GMD algorithm with parameter $P$. This $\operatorname{CGA}(P)$ processes all the vectors $\mathbf{w}=\mathbf{z}+\mathbf{e}$ with $e$ in $E$. Define the following set of integers:

$$
I(P)=\{i: 0 \leq i \leq d-2 P-1 \text { and } d-i \text { is odd }\} .
$$

For each $w$ and each integer $i \in I(P)$, erase $i$ symbols of $w$ starting from symbol position $P+1$ to symbol position $P+i$. This results in a vector $w^{*}$ with $i$ erasures. Perform errorand-erasure decoding on $w^{*}$. If decoding is successful, the decoded codeword is a candidate codeword. After performing

$$
q^{P}(\lfloor(d+1) / 2\rfloor-P)
$$


decodings, we obtained a set of candidate codewords. Among these candidate codewords, the one with the best metric is the decoded codeword. For $P=0, \operatorname{CGA}(0)$ is simply the GMD algorithm and for $P=\lfloor d / 2\rfloor, C G A(P)$ is simply the Chase-2 algorithm. It can be proved that the performance of CGA $(P)$ improves as $P$ increases $[9]$.

The computational complexity of CGA $(P)$ is between those of GMD and Chase-2 algorithms. However, for large $q$ and $d$, the number of decoding given by (2) is still very dis. couraging for practical applications. We may modified this algorithm for further reduction in computational complexity. For $0 \leq i<P$, compute the conditional probabilities $p(y, g)$ for every $g$ in $\operatorname{GF}(q)$. Let $A_{i}\left(q^{\prime}\right)$ denote the set of $q^{\prime}$ symbols in $\operatorname{GF}(q)$ that give the $q^{\prime}$ largest conditional probabilities $p\left(y_{i} \mid g\right)$. Let $E^{\prime}$ denote the set of test error patterns with nonzero components confined to the first $P$ positions and chosen from $A_{1}\left(q^{\prime}\right)$ for $0 \leq i<P$. There are $q^{\prime P}$ error patterns in $E^{\prime}$. In the modified $C G A(P)$ algorithm, we use the error pattern in $E^{\prime}$ to generate candidate codewords by decoding $w^{\prime}=2+\mathrm{e}^{\prime}$ with $\mathrm{e}^{\prime}$ in $E^{\prime}$ and $i$ erasures in $w^{\prime}$ starting from symbol position $P+1$. Denote this modified algorithm with $\operatorname{CGA}\left(P, q^{\prime}\right)$. The total number of algebraic decodings required by $\operatorname{CGA}\left(P, q^{\prime}\right)$ is $q^{\prime P}(\lfloor(d+1) / 2\rfloor-P)$. It is clear that for $P=0, \operatorname{CGA}\left(0, q^{\prime}\right)$ is still the GMD algorithm.

Consider the decoding $(31,25,7)$ RS code over $G F\left(2^{5}\right)$. Then $q=32$. Suppose we chose $\boldsymbol{q}^{\prime}=2$. For $P=0,1,2$ and 3 , the bit error and block error performances of $\operatorname{CGA}(P, 2)$ are shown in Figure 4(a). For comparison, the performance of pure algebraic decoding is also included. We see that $\operatorname{CGA}(P, 2)$ for $P=1,2$ and 3 outperforms GND algorithm $(P=0)$ and pure algebraic decoding. At $B E R=10^{-4}, C G A(3,2)$ achieves a $0.7 \mathrm{~dB}$ coding gain over GMD algorithm and a $0.9 \mathrm{~dB}$ coding gain over pure algebraic decoding at a cost of 8 decoding trials while GMD algorithm requires 4 decoding trials. The bit and block error performances of the $(255,223)$ NASA standard RS code over $G F\left(2^{8}\right)$ are shown in Figure $4(b)$.

$\operatorname{CGA}\left(P, q^{\prime}\right)$ will be used in our proposed concatenated turbo coding system for decoding outer code. 


\section{A Concatenated Turbo Coding System}

In this section, we describe the proposed concatenated turbo coding system in which the inner turbo decoding is performed in parallel mode as described in Section 2 and the outer RS code is decoded with both algebraic and $\operatorname{CGA}\left(P, q^{\prime}\right)$ algorithms.

The concatenated system is shown in Figure 5(a). The inner code is a turbo code with two identical $\left(n_{i}, k_{1}\right)$ block component codes $C_{i}$ and the outer code is an $\left(n_{0}, k_{0}\right)$ RS (or shortened RS) code $C_{0}$ over $\mathrm{GF}\left(2^{m}\right)$ with minimum distance $d$. For binary transmission, each code symbol in $\mathrm{GF}\left(2^{m}\right)$ is expanded into a binary $m$-tuple, called an $m$-bit byte. Two types of concatenations are proposed: two dimensional and three dimensional concatenations. Both encoding and decoding are carried out in two stages.

\subsection{Encoding}

An information sequence of $\lambda m k_{o}$ bits is segmented into a sequence of $\lambda k_{o} m$-bit bytes. Each $m$-bit byte is regarded as symbol in $\operatorname{GF}\left(2^{m}\right)$. This sequence of $\lambda k_{0}$ bytes is arranged as a $\lambda \times k_{\circ}$ array $U$ as shown in Figure 5(b), each column consists of $\lambda$ bytes and each row consists of $k_{0}$ bytes. At the first stage of encoding, each row of $U$ is encoded into a RS codeword in $C_{0}$. This results in $\lambda$ RS codewords arranged in a $\lambda \times n_{0}$ array, denoted $V_{1}$, as shown in Figure 5(c).

Consider the two dimensional concatenated coding scheme. Let $\delta$ be the integer such that $\delta k_{i}=\lambda m n_{0}$. The interleaver between the outer encoder and the inner encoder reads the array $V_{1}$ column by column and write row by row into $\delta \times k_{i}$ array, denoted $V_{2}$, in bit form as shown in Figure 5(d). Each column consists of $\delta$ bits and each row consists of $k_{i}$ bits. At the second stage of encoding, the array $V_{2}$ is encoded into a $\delta \times\left(2 n_{i}-k_{i}\right)$ array $V_{3}$ with turbo encoding as shown in Figure $5(e)$. The turbo encoding is carried out as described in Section 2. The array $V_{3}$ consists of three subarrays, the information array $V_{2}$, the parity 
arrays $P_{1}$ and $P_{2}$. Each row of $V_{3}$ is a lurbo codeword which consists of $k_{i}$ information bits and two parts of parity check bits, each consists of $n_{i}-k_{i}$ parity check bits. $V_{3}$ is a concatenated turbo codeword. $V_{3}$ is transmitted row by row. The rate of the concatenated turbo code is

$$
R=\frac{\lambda m k_{0}}{\lambda m n_{0}+2 \lambda m n_{0}\left(n_{i}-k_{i}\right) / k_{i}} .
$$

If a three dimensional concatenated coding scheme is used, an integer $\delta^{\prime}$ is chosen such that $\delta^{\prime} k_{i}=\lambda n_{0}$. The array $V_{1}$ is read column by column and write row by row into $m \delta^{\prime} \times k_{i}$ arrays, denoted $V_{2}^{[1]}, V_{2}^{[2]}, \ldots, V_{2}^{[m]}$, in bit form as shown in Figure $5(f)$ (bit demultiplexing). The $i$-th bit of each $m$-bit byle is put into array $V_{2}^{[1]}, 1 \leq i \leq m$. Al the second stage of encoding, each array $V_{2}^{(j)}$ is encoded into a $\delta^{\prime} \times\left(2 n_{i}-k_{i}\right)$ array $V_{3}^{(j)}$ with turbo encoding, $1 \leq i \leq m$. Each array $V_{3}^{(1)}$ also consists of three subarrays, the information array $V_{2}^{[3]}$, the parity arrays $\mathrm{P}_{1}^{[0]}$ and $\mathrm{P}_{2}^{[0]}$ as shown in Figure $5(\mathrm{~g})$. The three dimensional concatenated code has the same code rate as the two dimensional concatenated code.

\subsection{Decoding}

First, consider the two dimensional concatenated coding scheme. Let $\mathbf{R}$ be the received array corresponding to $V_{3}$. It is then decoded in two stages, the inner decoding and outer decoding. At inner decoding, $\mathbf{R}$ is decoded with turbo decoding in parallel mode as described in Section 2. At end of each phase of a decoding iteration, the two component decoders of the turbo decoder, DEC1 and DEC2, produce two decoded information arrays, $\hat{\mathbf{V}}_{2}^{(1)}$ and $\hat{\mathbf{V}}_{2}^{(2)}$, along with the reliability $L$-values of decoded bits and extrinsic values. The two estimated arrays $\hat{\mathrm{V}}_{2}^{(1)}$ and $\dot{\mathrm{V}}_{2}^{(2)}$ are compared. Using B.M stopping criterion, if $\hat{\mathrm{V}}_{2}^{(1)}$ and $\hat{\mathrm{V}}_{2}^{(2)}$ match in every bit position, the turbo decoding iteration process can be stopped.

If two corresponding bits do not match at a certain bit position in $\hat{\mathbf{V}}_{2}^{(1)}$ and $\hat{\mathrm{V}}_{2}^{(2)}$, a hard-decision at this position based on $L\left(\hat{u}^{(1)}\right)$ and $L\left(\dot{u}^{(2)}\right)$ given in $(1)$ is likely to result 
in an error. Suppose $\hat{V}_{2}^{(1)}$ and $\hat{V}_{2}^{(2)}$ do not match in all bit positions, we rearrange tbem into arrays $\hat{V}_{i}^{(1)}$ and $\dot{V}_{i}^{(2)}$ corresponding to the RS code array $V_{1}$ shown in Figure $5(c)$. The mismatched bit positions in $\hat{V}_{2}^{(1)}$ and $\hat{V}_{2}^{(2)}$ will result in mismatched symbol positions in $\hat{V}_{1}^{(1)}$ and $\hat{V}_{i}^{(2)}$. Now we compare the corresponding rows of $\hat{V}_{i}^{(1)}$ and $\hat{V}_{i}^{(2)}$ and check how many symbol positions where two corresponding symbols do not match. Hard decisions at these symbol positions are likely to result in symbol errors. If the number of mismatched symbol positions for each pair of corresponding rows in $\dot{V}_{1}^{(1)}$ and $\dot{V}_{i}^{(2)}$ is less than or equal to the error correcting capability $t=\lfloor(d-1) / 2\rfloor$ of the outer code and if symbol errors resulting from hard decisions are only confined in these mismatched positions, then the outer RS code can be used to correct these errors. Based on this reasoning, we now can formulate a criterion for stopping the inner turbo decoding iteration and let the outer decoder to remove the remaining errors (if any).

Symbol Matching (SM) Stopping Criterion: Compare row by row $\dot{\mathrm{V}}_{1}^{(1)}$ and $\hat{V}_{i}^{(2)}$. If the number of mismatched symbol positions for each pair of corresponding rows is less than or equal to $\lfloor(d-1) / 2\rfloor$, then stop the inner turbo decoding iteration.

When the inner turbo decoding is stopped based on the SM criterion, hard decisions are made at the mismatched positions in $\hat{V}_{1}^{(1)}$ and $\hat{V}_{1}^{(2)}$ based on the $L$-values $L\left(\dot{V}_{1}^{(1)}\right)$ and $L\left(\hat{\mathbf{V}}_{i}^{(2)}\right)$. This results in an estimated array $\mathbf{V}_{i}$. Then second decoding stage starts. The outer decoder decodes each row of $V_{i}$ based on an algebraic decoding algorithm, such as the Euclidean algorithm. If each row of $\mathbf{V}_{\mathbf{i}}$ is decoded successfully, decoding is done. The parity check symbols are removed from each decoded RS codeword and the decoded information symbols are then delivered to the user. If not all the rows of $V_{i}$ are decoded successfully, the outer decoder instructs the inner turbo decoder to resume decoding iteration from the phase where it was stopped. Then the above inner/outer decoding process continues until either all the rows of $V_{i}$ are decoded successfully or the inner decoding reaches a maximum 
number $I_{\max }$ of iterations. For the latter case, the outer decoder decodes $\mathrm{V}_{\mathrm{i}}$ based on a $\operatorname{CGA}\left(P, q^{\prime}\right)$ algorithm and stops.

The above decoding process is illustrated by the flow chart shown in Figure 6.

The SM criterion is a very effective stopping criterion. It requires only simple binary or logic operations. At the end of each decoding phase, it requires $2 K=2 \delta k_{i}$ binary operations to make bard decisions to form estimated arrays, $\hat{V}_{i}^{(1)}$ and $\hat{V}_{i}^{(2)}$, and $K$ bit-comparisons to compute the numbers of mismatched symbol positions for all pairs of corresponding rows in $\dot{V}_{i}^{(1)}$ and $\dot{V}_{i}^{(3)}$.

The decoding for the three dimensional concatenated code is similar to the two dimensional code. At the receiver, there are $m$ received arrays, $\mathbf{R}^{(1)}, \mathbf{R}^{(2)}, \cdots, \mathbf{R}^{[m]}$ corresponding to $V_{3}^{(1)}, V_{3}^{(2)}, \ldots, V_{3}^{(m)}$, respectively. It is also decoded in two stages, inner decoding and outer decoding. At inner decoding, each $\mathbf{R}^{(\mathfrak{l})}, 1 \leq i \leq m$, is decoded with turbo decoding individually. This allows us to use $m$ identical turbo decoders in parallel, each for decoding one received array, in order to speed up decoding process. At end of each phase of a decoding iteration for all turbo decoders, the SM stopping criterion is used to lerminate the iterative process of inner turbo decoding. When the S.M criterion is satisfied, bard decisions are made by all $m$ decoders. These hard decisions form $\lambda n_{0} m$-bit bytes such that the $i$-th bit of each byte is from the hard decision of the $i$-th decoder, $1 \leq i \leq m$. These $\lambda n_{0} m$-bit bytes form the estimated array $V_{i}$. Then, the second decoding stage starts as the same as the two dimensional concatenated code.

\section{Examples and Simulation Results}

The proposed interactive concatenated turbo coding system has been simulated for both AWGN and Rayleigh channels. Simulation results show that this coding system achieves both good bit-error and frame error performances without error foor. Furthermore, the SM 
stopping criterion effectively terminates the inner turbo decoding iteration and shortens the inner decoding delay.

Consider an example in which the $(228,212)$ shortened RS code over $G F\left(2^{8}\right)$ is used as the outer code and the $(64,57)$ distance-4 extended Hamming code is used as the two component codes for constructing the inner turbo code. We choose $\lambda=4$. Then $\delta=128$. The rate of this system is $R=0.75$. The bit-error and frame-error performances of the two dimensional scheme of this system for AWGN channel are shown in Figures 7(a). We see the waterfall performance without error foor. For the Rayleigh channel without channel side information, the bit-error and frame-error performances are shown in Figure 7(b). Again they display waterfall error performance. Form Figure $7(a)$, we see that for AWGN channel and at $B E R=10^{-4}$, the proposed iterative decoding achieves a $5.5 \mathrm{~dB}$ coding gain over the uncoded BPSK system which is only $1.2 \mathrm{~dB}$ away from the Shannon limit for rate $R=0.75$.

The bit-error performance of the three dimensional scheme of this system is also shown in Figure $7(\mathrm{a})$. From Figure $7(\mathrm{a})$, we see that the three dimensional concatenated coding scheme has better performance than the two dimensional scheme at low SNR and the gap between those two schemes is small at high SNR. In the three dimensional concatenated coding scheme, $m$ bits in each code symbol over $\mathrm{GF}\left(2^{m}\right)$ are decoded by $m$ turbo decoders independently. This results in a better error performance at low SNR compared with the two dimensional code. Furthermore, it allows us to use $m$ identical turbo decoders to decode $m$ received arrays in parallel which increases the decoding speed by a factor close to $m$.

To show the effectiveness of the SM criterion, we stop the inner turbo decoding iterations with four stopping criteria: (1) a fixed number of iterations $=10$, (2) the CE criterion, (3) the BM criterion, and (4) the SM criterion. The maximum number of decoding iterations, $I_{\max }$, for the last 3 criteria is also set to 10 . Tables $1(a)$ and $1(b)$ display: (1) the average numbers of iterations required for each criterion, and (2) the number of blocks in errors after inner decoding, outer algebraic decoding, and $\mathrm{CGA}(1,2)$ decoding of 1,000 blocks. Tables $1(\mathrm{a})$ and 
1(b) are for SNR's 2.9 and $3.0 \mathrm{~dB}$, respectively. Consider SNR=3.0 dB. From Table 1(b), we see that SM criterion saves 5.72 iterations while $B M$ and $C E$ criteria save 4.74 and 2.37 iterations, respectively, on average compared with $I_{\max }=10$. After inner decoding, if SM criterion is used, there are 147 RS words in error while using $C E$ criterion, there are only 12 RS words in error. However, after either algebraic or $\operatorname{CGA}(1,2)$ outer decodings, there is no RS word in error. Finally, Figure 8 displays the average numbers of decoding iterations required with $S M$ and $C E$ criteria, respectively, for the example system over AWGN channel. From Figures $3,7(\mathrm{a})$ and 8 , we see that SM criterion is more effective than the CE criterion. It reduces the number of decoding iterations with very little performance degradation.

Consider another example in which the $(255,223)$ RS code over $\operatorname{GF}\left(2^{8}\right)$ is used as outer code and the $(32,16)$ RM code with minimum distance 8 is used as the two component codes for constructing the inner turbo code. We choose $\lambda=4$, then $\delta=510$. The rate of this system is $R=0.29$. The bit-error and frame-error performances of the two dimensional scheme of this system for AWGN channel are shown in Figure 9. We see that at BER $=10^{-4}$, the proposed decoding achieves a $7.3 \mathrm{~dB}$ coding gain over the uncoded BPSK system which is $1.75 \mathrm{~dB}$ away from the Shannon limit for rate $R=0.29$.

\section{Conclusion}

In this paper, we have presented a high performance concatenated turbo coding system in which the inner and outer decoders interact to achieve good error performance. An effective criterion for stopping inner turbo decoding iteration has been proposed, which is more effective than the $\mathrm{CE}$ criterion in reducing the number of decoding iterations. Also presented in this paper is a reliability-based decoding algorithm which provides a good tradeoff between the error performance of Chase-2 algorithm and complexity of GMD decoding algorithm. 


\section{References}

(1) C. Berrou, A. Glavieux and P. Thitimajshima, "Near Shannon Limit Error-Correcting Coding and Decoding: Turbo Codes," Proc. of IEEE Inll. Conf. on Communications, Geneva, Switzerland, pp. 1064-1070 May 1993.

[2] S. Benedetto and G. Montorsi, "Unveiling Turbo Codes: Some Results on Parallel Concatenated Coding Scbemes," IEEE Trans. Inform. Theory, Vol. 42, pp. 409.428. March 1996.

[3] J. Hagenauer, E. Offer and L. Papke, "Iterative Decoding of Binary Block and Convolutional Codes," IEEE Trans. on Information Theory, Vol. 42, pp. 429-445, March 1996.

[4] D. Chase, "A Class of Algorithms for Decoding Block Codes with Channel Measurement Information," IEEE Trans. on Inform. Theory, Vol. 18, pp. 170-182, January $19 i 2$.

[5] G. D. Forney, Jr., "Generalized Minimum Distance Decoding," IEEE Trans. on Inform. Theory, Vol. 12, pp. 125-131, April 1966.

[6] D. Divsalar and F. Pollara, "Turbo Codes for PCS Applications," Proc. of ICC'95, Seattle, WA, June 1995.

[7] Y. Shao, M. P. C. Fossorier and S. Lin, "Two Simple Stopping Criteria for Iterative Decoding," IEEE Trans. on Communications, Vol. 47, pp. 1117-1120, August 1999.

[8] G. Einarsson and C. Sundberg, "A Note on Soft Decision Decoding with Successive Erasures," IEEE Trans. on Information Theory, Vol 22, pp. 88-96, January 1976. 
(9) H. Tang, Y. Liu, M. P. Fossorier and S. Lin, "On Combining Chase-2 and GMD Decoding Algorithms for Nonbinary Block Codes," Proc. of AAECC.19, Hawaii, November 1999, and submitted to IEEE Communication letters, September, 1999. 
Table 1: Comparisons of different stopping criteria

\begin{tabular}{|c|l|c|c|c|c|}
\hline \multicolumn{2}{|c|}{} & iterations $=10$ & CE criterion & BM & SM $\leq t$ \\
\hline \multirow{2}{*}{$\begin{array}{c}\text { block errors } \\
\text { per } 1000 \\
\text { sequences }\end{array}$} & turbo decoding & 20 & 23 & 27 & 178 \\
\cline { 2 - 6 } & alg. decoding & 12 & 14 & 14 & 19 \\
\hline \multicolumn{2}{|c|}{ average iteration number } & 10 & 8.05 & 6.24 & 5.10 \\
\hline
\end{tabular}

(a) $S . N R=2.9 \mathrm{~dB}$

\begin{tabular}{|c|l|c|c|c|c|}
\hline \multicolumn{2}{|c|}{} & iterations $=10$ & CE criterion & $\mathrm{BM}$ & $\mathrm{SM} \leq t$ \\
\hline \multirow{2}{*}{$\begin{array}{c}\text { block errors } \\
\text { per } 1000\end{array}$} & turbo decoding & 10 & 12 & 15 & 147 \\
\cline { 2 - 6 } sequences & alg. decoding & 0 & 0 & 0 & 0 \\
\cline { 2 - 6 } & $\mathrm{CGA}(1)$ & 0 & 0 & 0 & 0 \\
\hline \multicolumn{2}{|c|}{ average iteration number } & 10 & 7.03 & 5.26 & 4.28 \\
\hline
\end{tabular}

(b) $S . N R=3.0 \mathrm{~dB}$

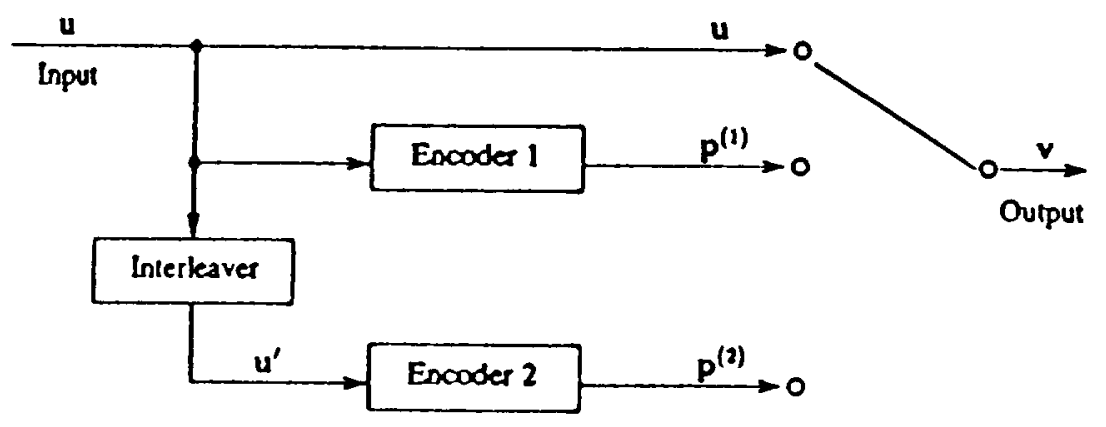

Figure 1: A block diagram of the turbo encoder with two component codes 


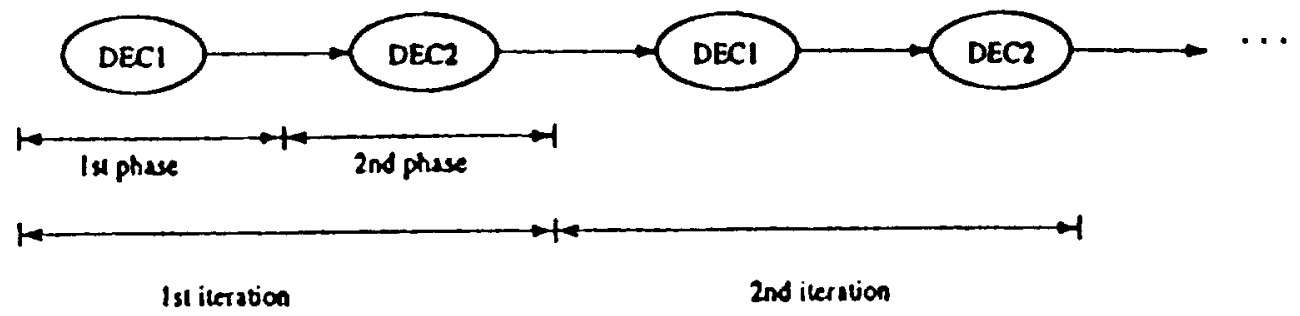

(a) Serial mode

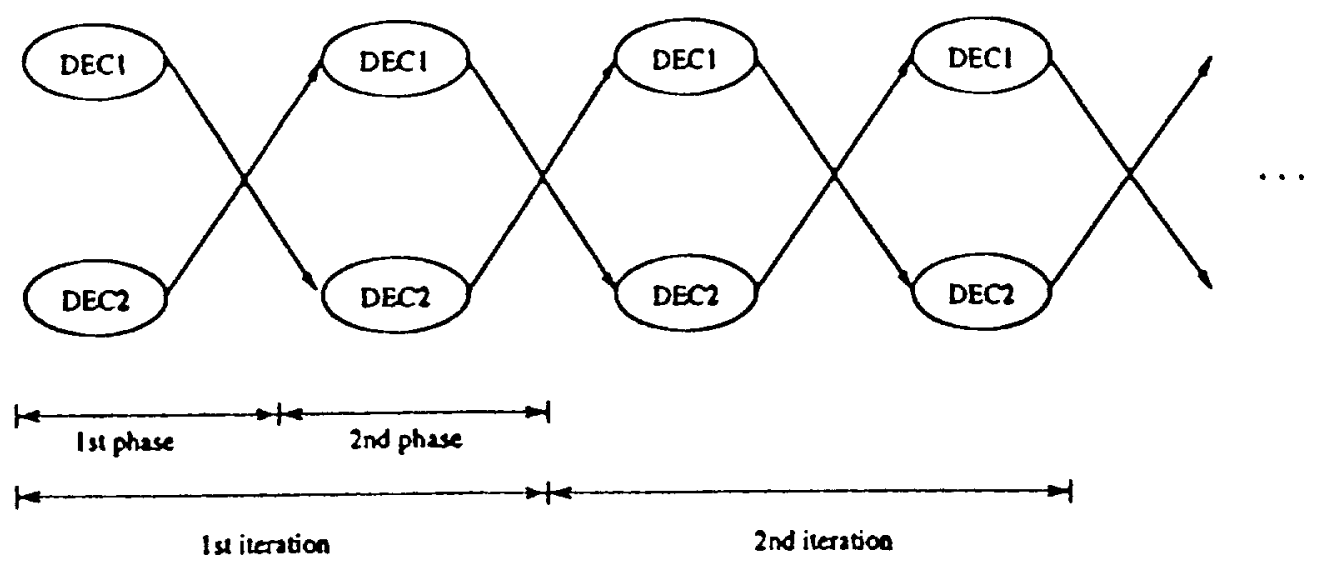

(b) Parallel mode

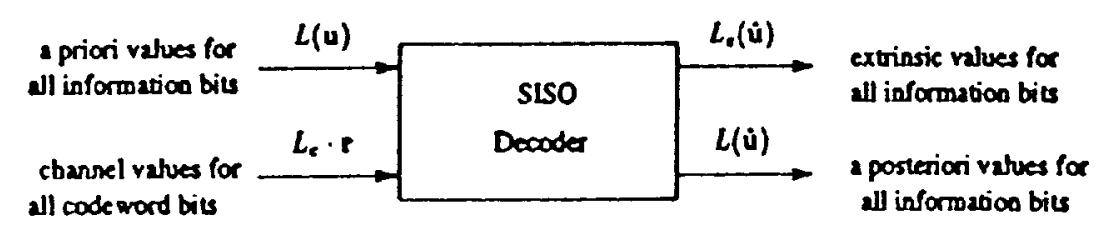

(c) DEC1 or DEC2

Figure 2: Serial and parallel decoding structures of turbo codes 


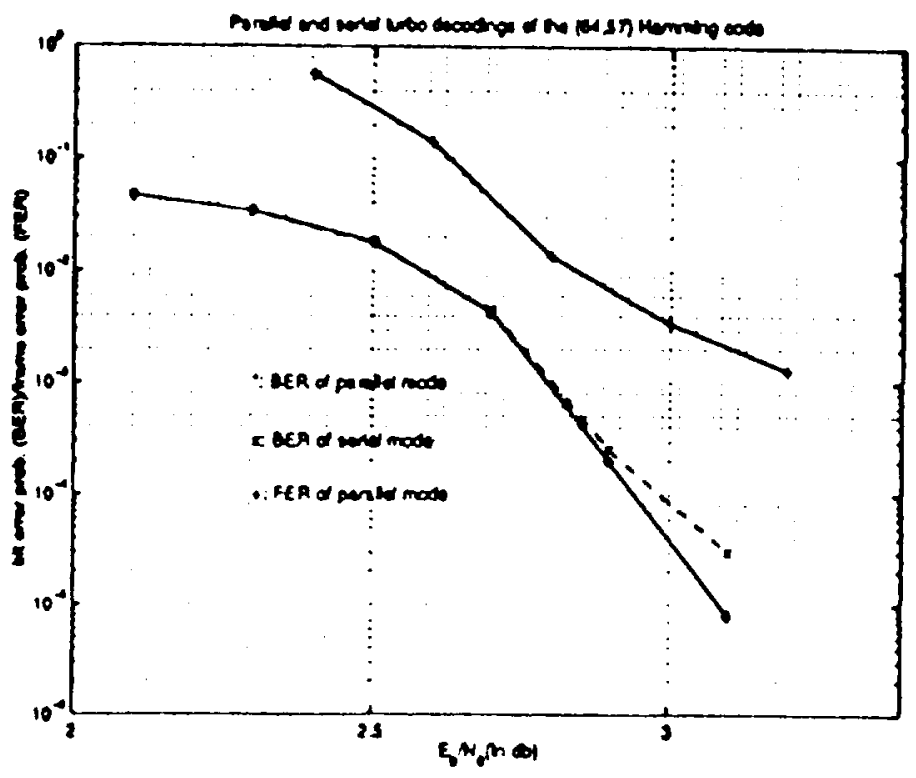

(a)

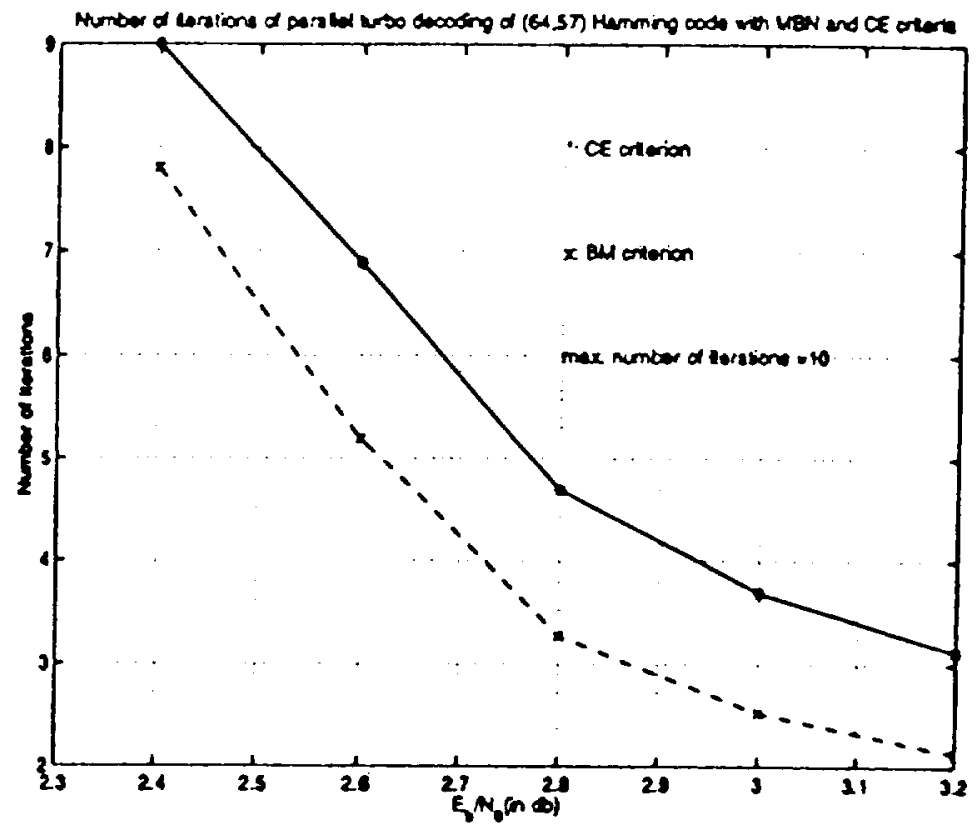

(b)

Figure 3: Error performances and numbers of iterations of parallel and serial turbo decodings of the $(64,57)$ Hamming code 


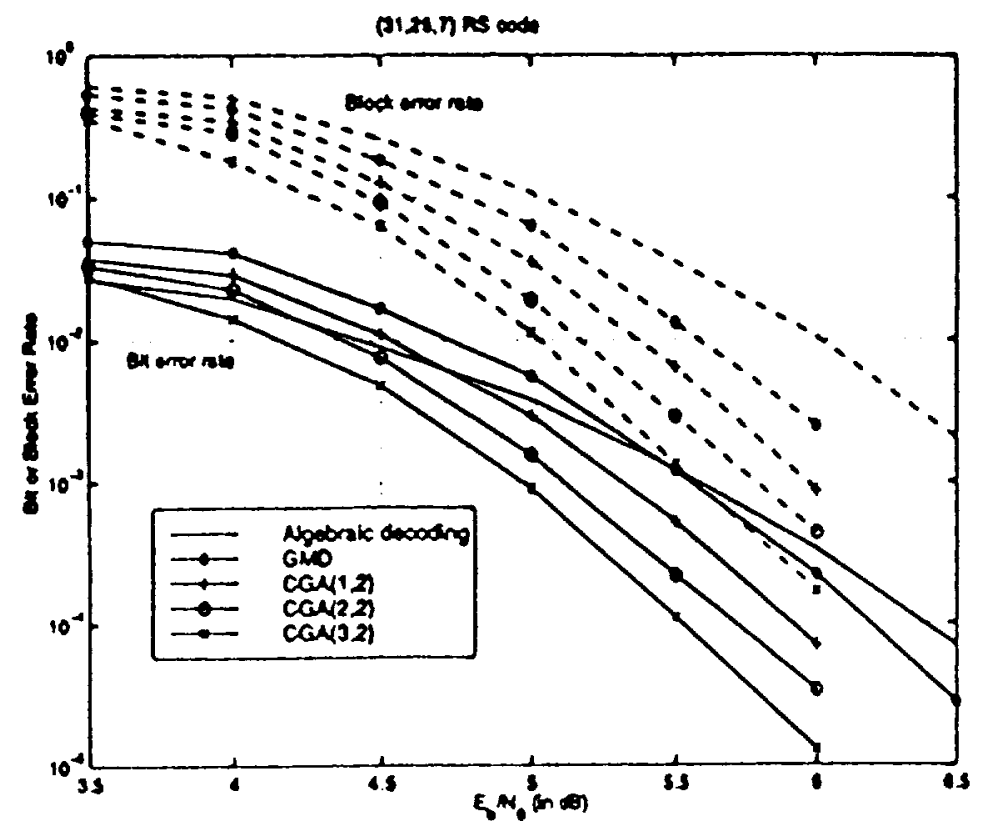

(a)

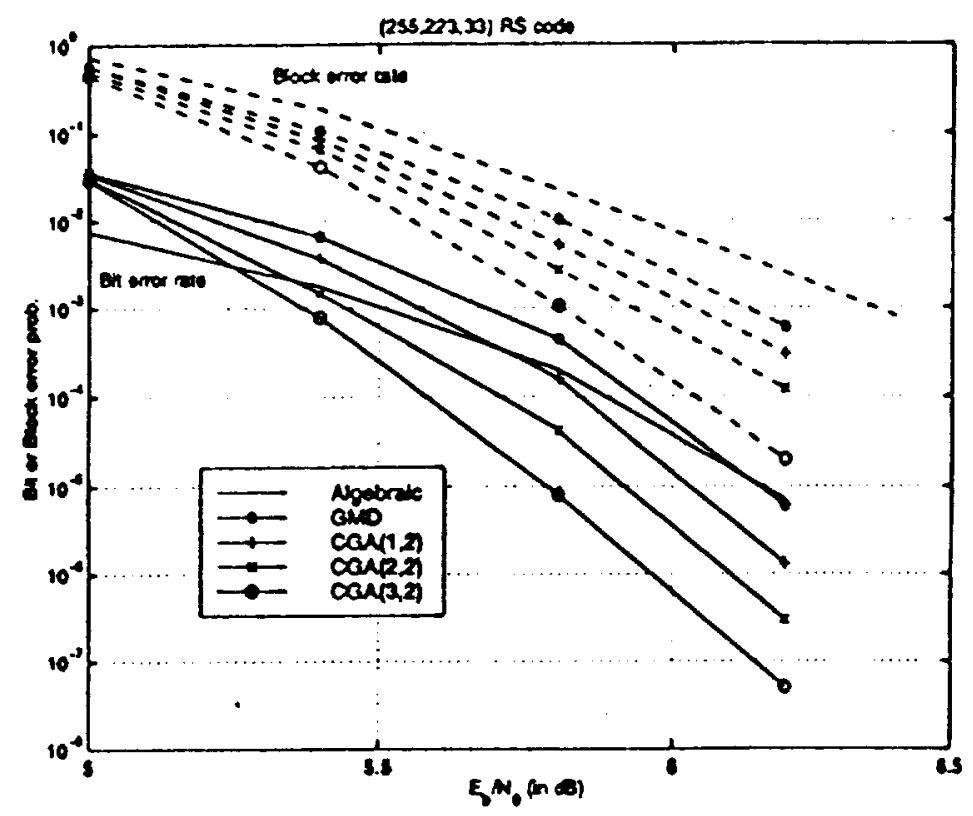

(b)

Figure 4: Bit and block error performances of Chase-GMD decoding of the $(31,25,7)$ and $(255,223,33)$ RS codes 


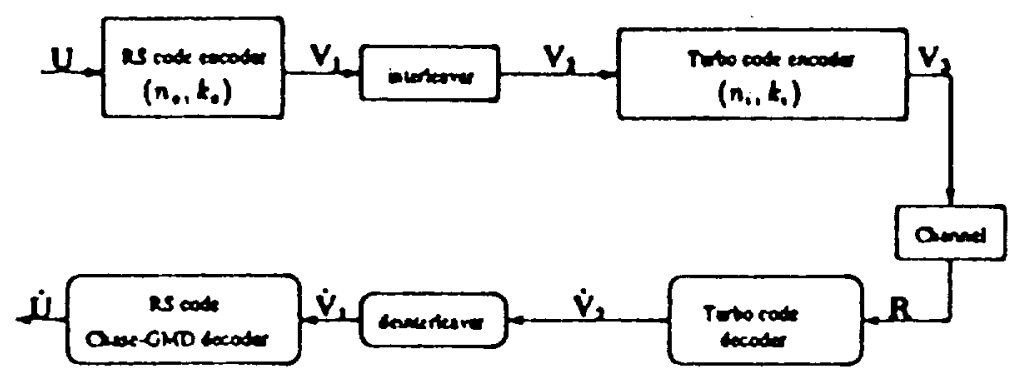

(a) Block diagram of a concalenaled wrto cosing systero

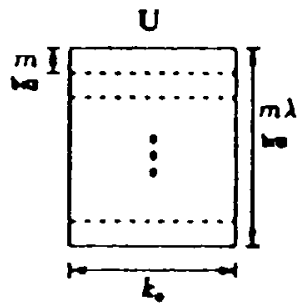

(b)

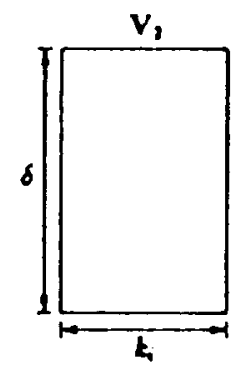

(d)

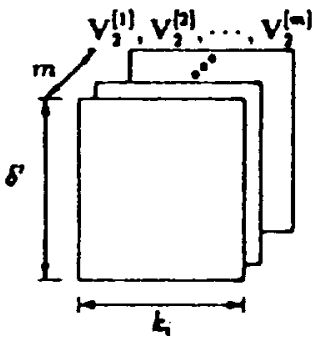

(ก)

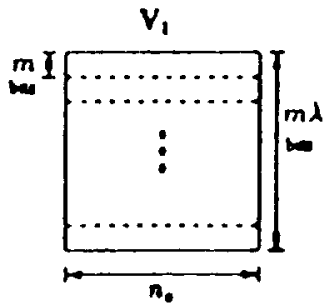

(c)

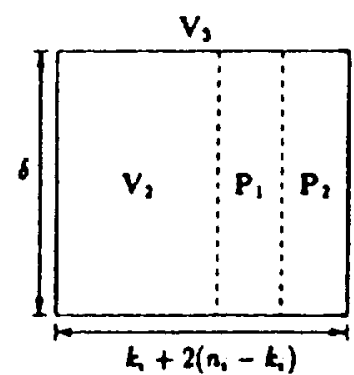

(c)

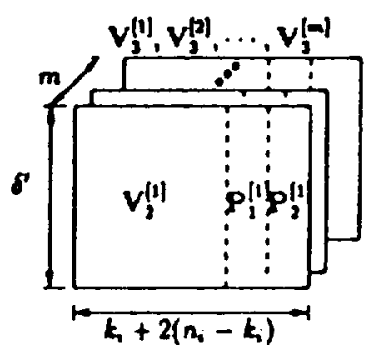

(8)

Figure 5: Two and three dimensional concatenated turbo codes 


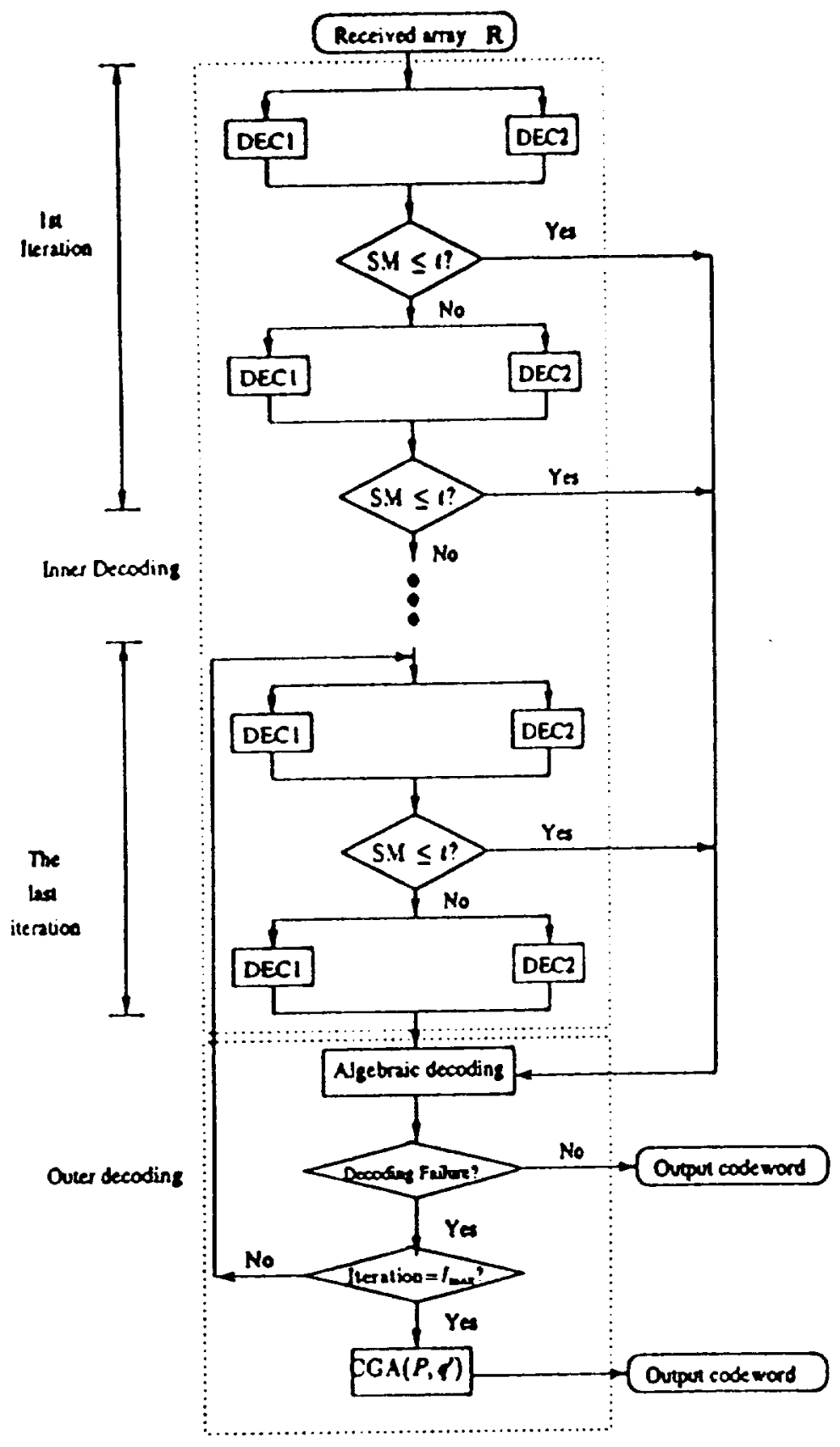

Figure 6: Flow chart for iterative decoding of concatenated turbo codes 


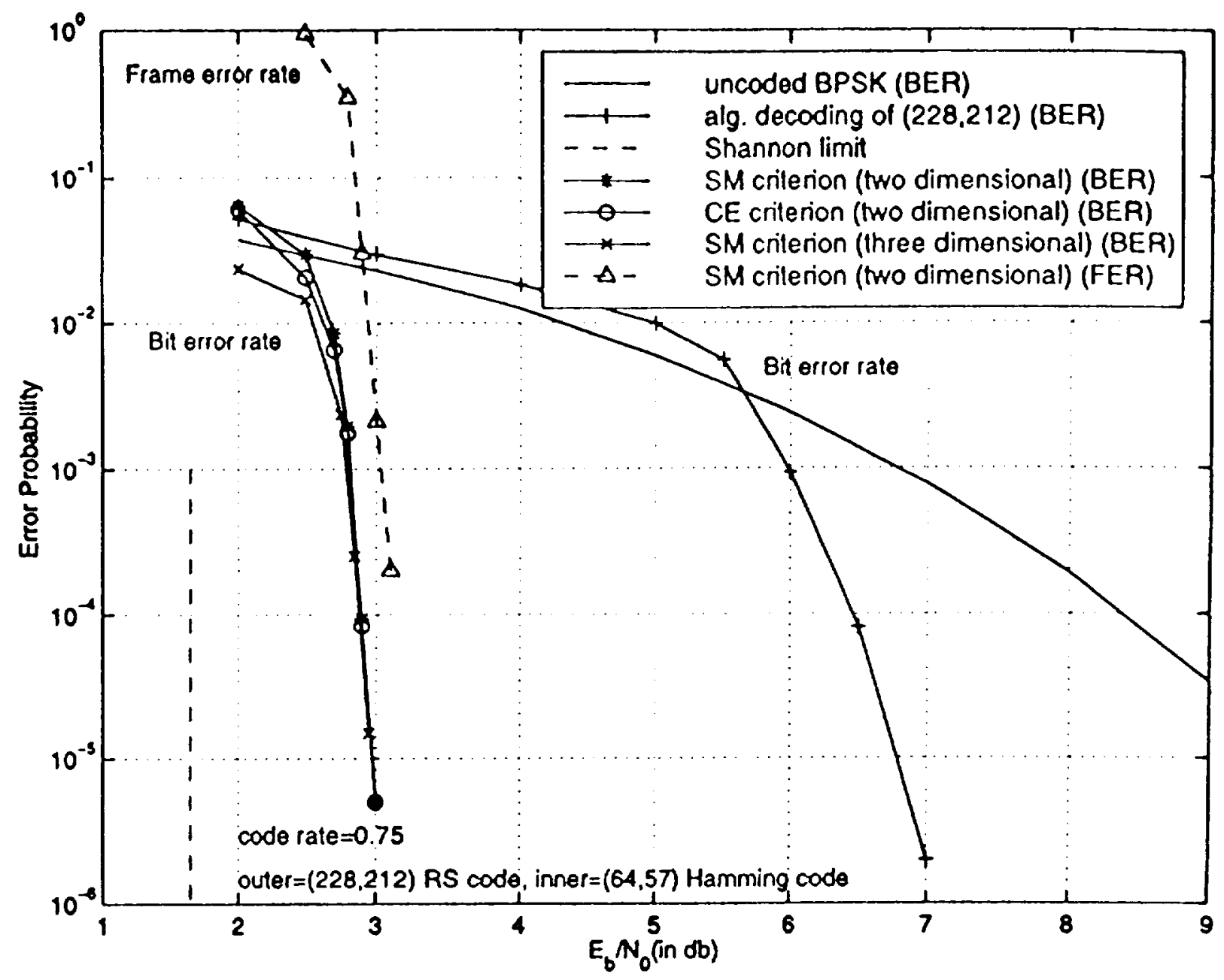

Figure 7: (a) Bit and frame error performances of iterative decoding of the concatenated turbo code with the $(228,212)$ shortened RS code and the $(64,57)$ extended Hamming code as outer code and component codes of inner turbo code over AWGN channel 


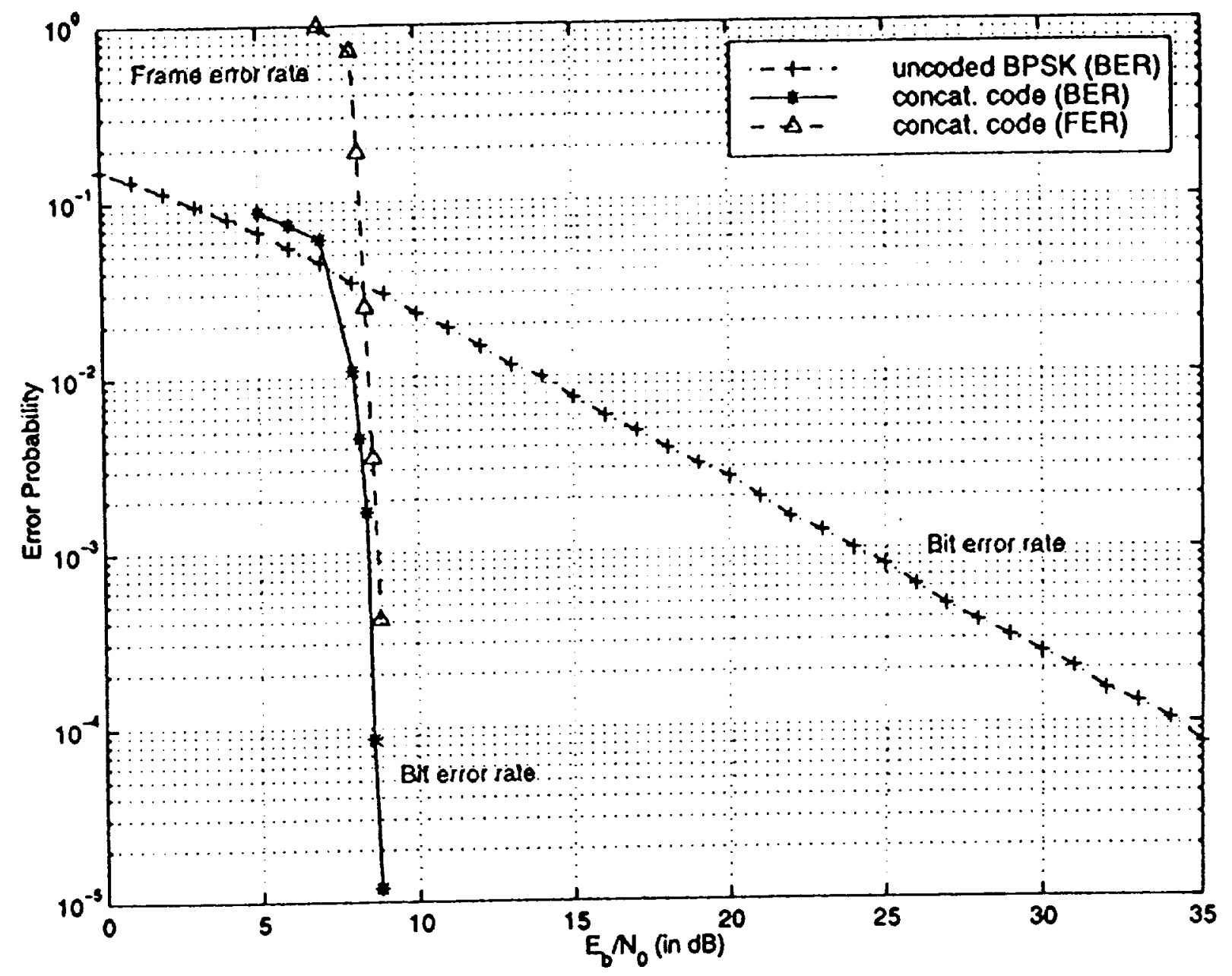

Figure 7: (b) Bit and frame error performances of iterative decoding of the concatenated turbo code with the $(228,212)$ shortened RS code and the $(64,57)$ extended Hamming code as outer code and component codes of inner turbo code over Rayleigh fading channel 


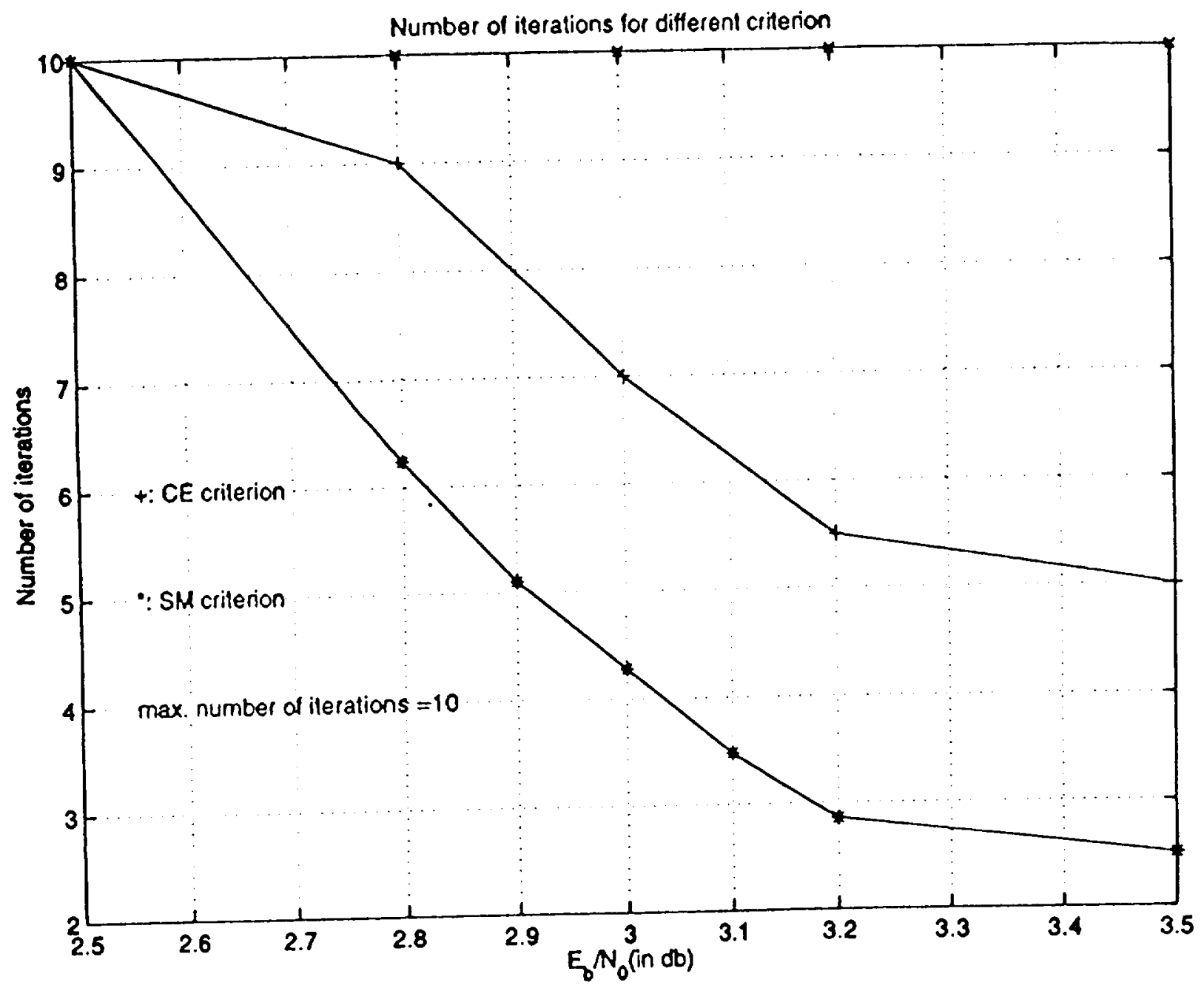

Figure 8: Number of iterations of SM and CE criteria of the concatenated turbo code with the $(228,212)$ shortened RS code and the $(64,57)$ extended Hamming code as outer code and component codes of inner turbo code over AWG.N channel 


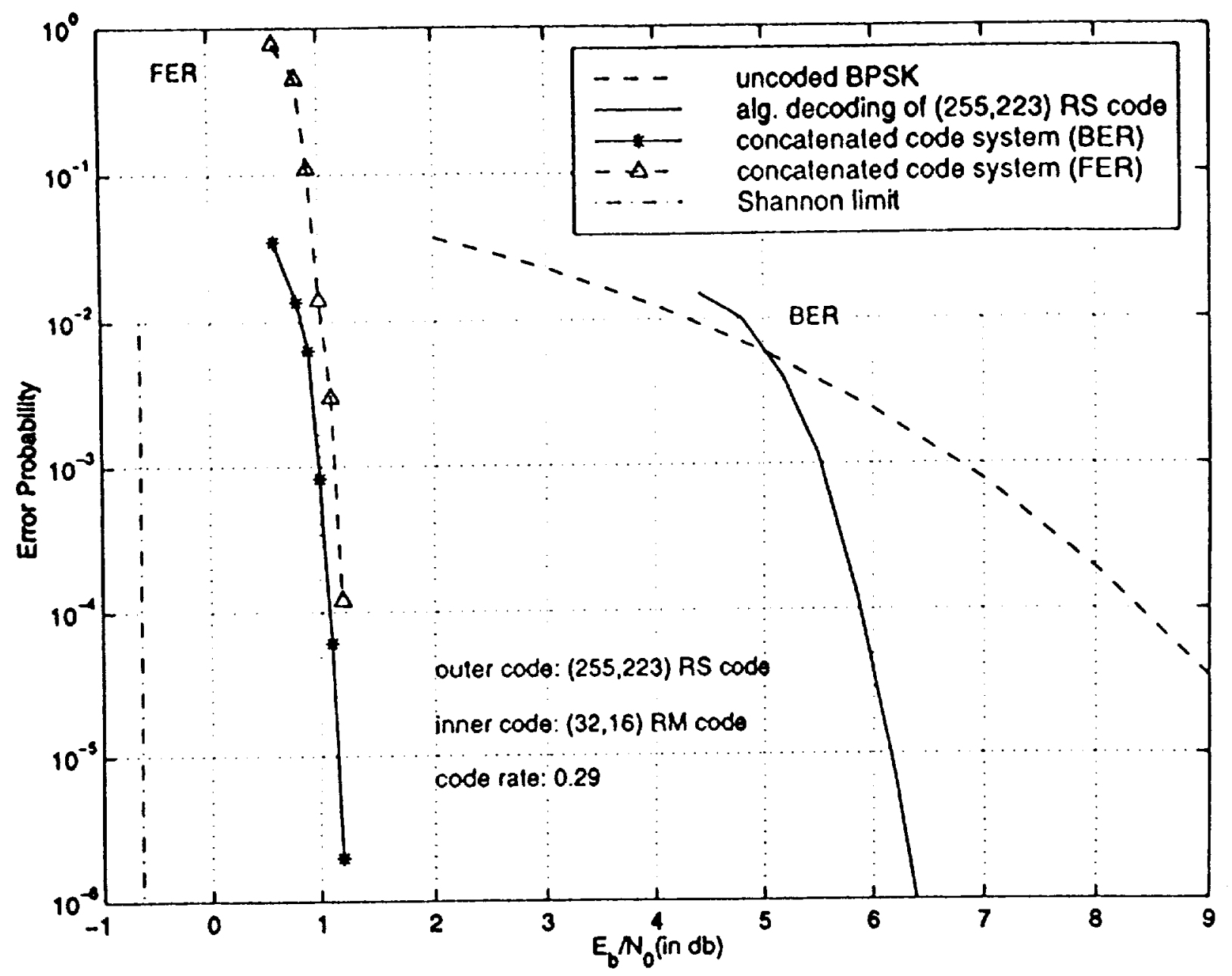

Figure 9: Bit and frame error performances of iterative decoding of the concatenated turbo code with the $(255,223)$ RS code and the $(32,16)$ RM code as outer code and component codes of inner turbo code over AWGN channel 


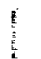


